\title{
"Use of an Arabic-language Version of TOPL-2 to Identify Typical and Atypical Manifestations of Pragmatic Language Impairment in Individuals with Developmental Dysphasia"
}

\author{
Ahmed Mohammed S. Alduais ${ }^{1}$, Fayza Saleh Al-Hammadi ${ }^{2}$, Rasha Mohammed \\ A. Shoeib ${ }^{3}$, Khalid Hassan Al-Malki ${ }^{4}$, Farah Hameid Alenezi ${ }^{5}$ \\ ${ }^{\text {I}}$ (Research Chair of Voice and Swallowing Disorders, College of Medicine/ King Saud University, Kingdom of \\ Saudi Arabia) \\ ${ }_{2}^{2}$ (Department of English Language, College of Arts/ King Faisal University, Kingdom of Saudi Arabia) \\ ${ }_{3}^{3}$ (Communication and Swallowing Disorders Unit (King Abdulaziz University Hospital), College of Medicine/ \\ King Saud University, Kingdom of Saudi Arabia) \\ ${ }^{4}$ (ENT Department, College of Medicinel King Saud University, Kingdom of Saudi Arabia) \\ ${ }^{5}$ (English Language Department, College of Arts/ King Saud University, Kingdom of Saudi Arabia)
}

\begin{abstract}
Purpose: To identify typical and atypical pragmatic language impairment PLI manifestations and determine the strengths and weaknesses of different types of pragmatic skills using an Arabic version of Test of Pragmatic Language TOPL-2.

Methods: Twelve individuals (F: 5, M: 7) clinically diagnosed with different types of developmental dysphasia $D D-S L I(n: 6), D L D(n: 4), L D(n: 1)$, and (DLAPh: 1) between 6 and 12 years old took an Arabic version of the TOPL-2. The test was administered twice to assure the consistency of the elicited data.

Results: The results of the doubled-administration of the Arabic TOPL-2 are somewhat but not completely consistent. Participants manifested more atypical PLI manifestations than typical ones. There might be an intricate relationship between pragmatic competence $P C$ and Intelligence quotient IQ test performance. The participants' PC levels did not correspond to their real ages and grades, which affected their school achievement and social lives. Finally, PLI severity seems to be controlled by the primary disorder type: congenital (developmental-dysphasia), biolinguistic (genetic) or neurolinguistic (acquired child aphasia).

Conclusions: The used psycholinguistic marker, the Arabic TOPL-2, could give a proposed order for the most atypical PLI manifestations and many typical ones
\end{abstract}

.Keywords - Arabic TOPL-2 version, clinical treatment plans, developmental dysphasia, typical and atypical manifestations of pragmatic language impairment.

\section{INTRODUCTION}

Language operates at two basic analytical levels, concrete and abstract, which can also be represented by parallel linguistic items and in terms of being phonemic (phoneme) or phonological (allophone), morphological (morpheme) or lexical (allomorph), a sentence (written) or an utterance (spoken), semantic (linguistic meaning) or pragmatic (implied/intended meaning(s)), grammatical (well-formed structures) or acceptable (may have ill-formed structures but is acceptable) and based on competence (linguistic knowledge in the mind) or on performance (actions of performing the stored linguistic knowledge in mind).

While linguistic competence LC refers to the ability of a person to acquire linguistic elements (phonetic, phonological, morphological, syntactic and semantic) and linguistic competence indicates the realworld use of these elements, communicative competence $\mathrm{CC}$ is the ability to use appropriate language elements in daily life communication, [1]. An individual may be linguistically competent but not communicatively competent. When a child fails to decide what to say to mean what and when to say to indicate what, $\mathrm{s} / \mathrm{he}$ is considered communicatively incompetent despite knowing what to say. Clearly, this applies more to pragmatics and is responsible for appropriate language use in context.

Pragmatic competency PC is widespread among children and adolescents as a sign or symptom of another disorder or as a disorder itself. A general identification of the problem, however, is not enough to treat it. Specific and detailed identification of the problem's typical and atypical PLI manifestations are needed, especially when starting a rehabilitation program or treatment plan for an individual who has been identified as pragmatically impaired. General evaluating tools cannot be used to evaluate or draw conclusions about an individual with PLI. This paper considers this issue, arguing that detailed and specific results about an individual with PLI are needed to build and design an effective rehabilitation program and treatment plan. 
In other situations, as in Speech and Language Pathology SALP, receptive and expressive language skills are more commonly used than in LC and CC. When talking about children with language and speech disorders, the former LC refers to the ability to understand what is being communicated and the latter CC to what should be communicated, [2,3]. A child who fails to receive communication elements, express them or act towards them has a problem that must be identified and assessed to determine a treatment plan.

Pragmatics, mainly PC, refers to the ability to communicate appropriately, use context-appropriate language and be able to say what in when and where. According to the American Speech-Language-Hearing Association (ASHA), [4], PC involves three major communication skills: using language, changing language and following rules. Table 1 summarized the above mentioned points regarding PC.

TABLE 1: Major Communication Skills of Pragmatic Competence

\begin{tabular}{lll}
\hline Using language & Changing language & Following rules \\
\hline Greeting (e.g., Hello) & $\begin{array}{l}\text { Used according to the needs of a } \\
\text { listener or situation }\end{array}$ & $\begin{array}{l}\text { Used for conversations and } \\
\text { storytelling }\end{array}$ \\
$\begin{array}{ll}\text { Talking differently to a baby than } \\
\text { to an adult }\end{array}$ & $\begin{array}{l}\text { Taking turns in conversation } \\
\text { Giving background information } \\
\text { a cookie.) }\end{array}$ & $\begin{array}{l}\text { Introducing topics of } \\
\text { conversation }\end{array}$ \\
$\begin{array}{l}\text { Demanding (e.g. Give me a } \\
\text { cookie.) }\end{array}$ & $\begin{array}{l}\text { Speaking differently in a } \\
\text { classroom than on a playground }\end{array}$ & $\begin{array}{l}\text { Staying on topic } \\
\text { Rephrasing when misunderstood }\end{array}$ \\
$\begin{array}{ll}\text { Promising (e.g., I am going to get } \\
\text { you a cookie.) }\end{array}$ & & $\begin{array}{l}\text { Using verbal and non-verbal } \\
\text { signals }\end{array}$ \\
$\begin{array}{l}\text { Requesting (e.g., I would like a } \\
\text { cookie, please.) }\end{array}$ & & $\begin{array}{l}\text { How close to stand to someone } \\
\text { when speaking }\end{array}$ \\
& & $\begin{array}{l}\text { Using facial expressions } \\
\text { Using eye contact }\end{array}$ \\
\hline
\end{tabular}

TOPL-2, a battery measuring the ability to use language in social interactions (pragmatics), measures six sub-components of pragmatics: physical setting, audience, topic, purpose, visual-gestural cues and abstraction. According to the authors of this test, 'it was standardized on a sample of 1,016 children residing in 21 states using gender, residence, race, geographic region and ethnicity as variables', [1 p. v]. This evaluation instrument contains a TOPL Booklet, black-and-white Picture Book and Examiner's Manual. Furthermore, both Phelps-Terasaki and Phelps-Gunn have adapted Norris and Hoffman's [5] pragmatic language model [Norris and Hoffman] designed and proposed this model as 'a framework for conceptualizing language in school-aged children' [1 p. 2] to create their own models in the second edition of TOPL. Table 2 illustrates both models. The S-D-S model is Norris and Hoffman's, and the S-D-S model with the addition of subcomponents is PhelpsTerasaki \& Phelps-Gunn's adapted model.

TABLE 2: Pragmatic Language Ability Components

\begin{tabular}{lll}
\hline Situational context & Discourse context & Semantic context \\
\hline Physical context & Topic & Visual-gestural cues \\
Audience & Purpose & Abstractions \\
& & Pragmatic evaluation \\
\hline
\end{tabular}

Human language develops gradually throughout childhood and then decays throughout old age. This parallel process can be interrupted when a child experiences delayed language development DLD or appears abnormal compared to peers or siblings. This study investigates DLD.

When treating persons with PLI(s), the provider must determine whether the patient has a brain injury. After this information is obtained, the preliminary treatment process can begin. First, the clinician, speech pathologist, psycholinguist, neurolinguist or clinical linguist assesses the person's general language ability to determine the PLI type. Second, a rehabilitation program is suggested and planned to improve the patient's pragmatic skills [6$10]$.

Kim investigates the characteristics of the language and pragmatic and social skills of twenty-two children with attention-deficit hyperactivity disorder and compares the outcomes of the tests, including the TOPL pragmatic language skill test, used to assess the subjects. It is concluded that children with ADHD demonstrated 'lower social skills than normally developing children' [11(p. 2)]. Kim also concluded that the TOPL results could not noticeably distinguish the strengths and weaknesses of children with attention deficit hyperattention disorder ADHD when compared to normally developing children [12].

Adams accounted for PLIs, described formal tests that can evaluate and assess persons with PLIs and published checklists for evaluating and assessing PLIs. TOPL and CELF have been highlighted here from 
among other formal and informal tests used to assess and identify PLIs in both children and adults [13]. Adams claimed that a core test of pragmatics can be decided in terms of the proliferation of the instruments used in the test [13].

Adhering to established guidelines and systematic action are necessary for a logical and reasonable diagnosis, evaluation and treatment. Busari \& Weggelaar, in their clinical review, discuss how to deal with children who are slow to speak and have delayed speech production and/or recognition. More importantly, it conveys how to assess a child with a development language delay (steps), evaluate him/her and report the results. They recommend that, when evaluating children with DLDs, one should 'carefully analyse the child's cognitive strengths and weaknesses' [14(p.272)].

Identifying, measuring and assessing PLIs are not sufficient. A researcher must also attempt a rehabilitation program or intervention guidelines based on the results. Ingersoll, Dvortcsak, Whalen, \& Sikora supported using the developmental social-pragmatic intervention model to teach children and adults with pragmatic or communication language disorders. The effectiveness of this model was tested on three children. The results indicate that such a model should be promoted and recommended for teaching social-communication skills to children and adults with autism and other relevant syndromes [15].

It would be unreasonable and illogical to explore PLI(s) from one perspective using a formal or informal test while ignoring other factors. Along these lines, Volden, Coolican, Garon, White, \& Bryson argued that other interrelated factors to both pragmatic language deficits (PLDs) or PLIs and autistic spectrum of disorders ASDs can be predicted and investigated. Such factors, however, contribute to the degree of severity and other issues when identifying, assessing and treating children and adults with ASDs and PLDs $[16,17]$.

Using the Arabic TOPL-2 as a psycholinguistic marker battery can thus help identify typical and atypical PLI manifestations in individuals with DLD. This ability of identification by itself supports the claim that determining the strengths and weaknesses of an individual with a PLI would help determine the exact level of a pragmatically impaired individual compared to others who are pragmatically impaired but with different primary disorders or causes. This would also result in a more effective rehabilitation program or treatment plan.

\section{II.I. Sampling}

\section{METHOD}

All participants in this study had primary cognitive disorders (delayed language disorders); their disorders differed but all exhibit a PLI as an accompanying symptom. All participants were Saudi nationals, diagnosed as having either a cognitive or linguistic disorder and brought to the Communication and Swallowing Disorders Unit to be treated by a communication disorders specialist.

The study used stratified sampling to select cases for this study; therefore, 12 abnormal children and adolescents took the Arabic TOPL-2 test twice each to test the consistency of the inferred judgments and outcomes of the research.

The setting of this research was the Communication and Swallowing Disorders Unit (CSDU), Research Chair of Voice, Swallowing, and communication Disorders, King Abdulaziz University Hospital (KAUH), College of Medicine, King Saud University, Riyadh, Kingdom of Saudi Arabia (2010-2011). A research proposal was also submitted to the IRB (Institutional Reviewing Board) of the Research Centre, King Khalid University Hospital, College of Medicine, King Saud University, Riyadh and approved. Table 3 shows the characteristics of the study participants.

TABLE 3: Characteristics of the participants

\begin{tabular}{ll} 
Variable & Characteristics \\
\hline No. of participants & 12 \\
Gender & F: 5- M: 7 \\
Age rang & $6-12$ \\
IQ range (verbal) & $54-110$ \\
Diagnosis & SLI: 6, DLD: 4, LD:1, and DLAPh:1 \\
Mother tongue lang. & Arabic \\
Used dialect & Saudi dialect \\
Nationality & Saudis \\
\hline
\end{tabular}

SLI: specific language impairment, DLD: delay language development, LD: learning disability, DLAPh: delay language affecting phonology

Finally, this study aimed to identify typical and atypical PLI manifestations in individuals with DLDs using the Arabic TOPL-2. The participants in this study suffer from different primary disorders with a PLI as a common symptom. Each group with a certain primary disorder clearly showed somewhat consistent outcomes regarding impaired and intact elements of the PC. Though few samples have been included in this study, the results can be compared with further research and generalized for similar samples under similar conditions. 


\section{II.2. Measures}

The TOPL-2, a common psycholinguistic marker tool, was used to measure the participants' PC. More specifically, this study used the Arabic TOPL-2.

TOPL-2 contains 43 items and 17 additional items. It targets three main pragmatic skill components, which also have the indicated sub-components: situational (physical context and audience), discourse (topic and purpose) and semantic contexts (visual-gestural cues, abstractions and pragmatic evaluation). The pragmatic evaluation subcomponent applied to the 17 additional items. The test was constructed to assess children and adolescents 6-18 years old and individuals whose PC interferes with their lives, including learning disability, language disorders, mental disorders, attention and/or emotional disorders . This test was first published in 1992, and a modified version (TOPL-2) was published in 2007. The authors of this test are Diana Phelps-Terasaki and Trisha Phelps-Gunn.

The TOPL-2 was translated into Arabic by Alduais et al. The researcher attempted to produce a usable, feasible, valid and reliable Arabic TOPL-2 that meets the same objectives as the original. The Arabic TOPL-2 has exactly the same format as the original TOPL-2 except for some internal changes based on cultural differences $[18,19]$.

Nominal-level measurement, where each variable has only numeric variables, was primarily used in this work. However, ordinal-level measurement was also used for variables, including descriptive ratings for each case and intelligence quotient IQ test results. The variables are generally discrete. The study is naturally qualitative, although it includes quantitative values and measurements in some situations to provide results that are more accurate.

Other secondary measurements used in this study include the following: 1) case report diagnoses (which disorders, including but not limited to linguistic disorders, have been identified?); 2) causes (according to the medical file reports, what are the causes of these disorders?); 3) recommendations or treatment plans (what plan or recommendations were suggested for treatment?); 4) case status (how has the case status progressed and when was the last time the patient reported to the clinic?); 5) social family status (has the doctor considered the social family status, i.e., the patient's relations with family members and acquaintances, and, if so, what relevant information is contained in the medical records?); and 6) IQ test results.

All types of reliability were considered. For inter-rater reliability, for instance, the booklets collected from the participants in this study were scored by three researchers who were trained to score the Arabic TOPL2. The scoring of this exam was identical to that of the original TOPL-2: a participant is awarded one point for a correct answer and none for an incorrect answer. The three researchers were given the possible correct and incorrect answers in Arabic. The inter-rater reliability for scoring the Arabic TOPL-2 items ranged between .96 and .97 . The test-retest reliability was estimated at .73 with a significance of .007 , which is significant at the 0.01 level,. Similarly, the internal consistency reliability was (.90) according to the study testing usability of the Arabic TOPL-2 [19-20].

This study carefully examined validity (construct validity). Considering the overall translation validity, both the face and content validities of the Arabic TOPL-2 are high [19]. Similarly high validities have been found for the concurrent validity (.24) with weakly correlated variables, convergent validity (.42) for the PP (CELF-4 subtest assessing pragmatic competency PC, weakly correlated validity, and (-.42) for the ORS (CELF-4 subtest assessing PC) and discriminant validity (.50) with moderately correlated variables [19-20].

\section{II.3. Design}

A non-experimental repeated test design was used in this study. This design can be depicted in the following notational parallel form:

$\mathrm{R}^{1} \mathrm{O} X-$

$\mathrm{R}^{2} \mathrm{OX}-$

$\mathrm{R}=$ randomized group, where superscripts indicate the same group, replication logic strategy

$\mathrm{O}=$ the one measure used in this study

$\mathrm{X}=$ non-treatment study

A randomized group of children and adolescents with various mental, emotional and language disorders that share a PLI, took the Arabic TOPL-2 twice to achieve the replication logic strategy.

This study is a multiple-case study, observational, descriptive, explanatory, and evaluative. Yin states that internal validity is 'inapplicable to descriptive or explanatory studies (whether the studies are case studies, surveys or experiments) which are not concerned with making casual claims' [21, (p. 36)]. The internal validity issue is thus inapplicable to this study. 


\section{II.4. Procedures}

Data-collection: The participants were met on their visits to the hospital and selected based on the applicability of the attained disorders to the purposes of the study (semi-parallel procedure).

Authenticity: The research proposal plan was first submitted to the College of Medicine's research center to be reviewed by the IRB for ethical issues. The board issued an approval letter, and the research center issued a consent form, which was handed to the parents of each child and/or adolescent who participated in the study. The paper assured confidentially and sensitivity of the collected data, which was for research purposes only. The same procedures were followed when issuing the consent form, which was issued and handed to the Research Chair of Voice, Swallowing, and Communication Disorders in the Communication and Swallowing Disorders Unit (CSDU), King Abdul-Aziz University Hospital (KAUH), King Saud University. The signed form allowed access to the archival records and medical reports of the participating cases and granted permission for interviews.

Test administration: The Arabic TOPL-2 was administered twice (replication logic strategy) in two sessions for those 6-7 years old and one session for those 8-18 years old. When critical situations for any case were observed or requested, pauses were given, and in some cases, the test administration was delayed to another day. The test was administered for the second time in the same way, one week after its first administration.

Test time and environment: Administering the test took approximately 1 to $1 \frac{1}{2}$ hours. A quiet room and a comfortable chair and table allowed the participant to look at the pictures and created a suitable test administration environment. All other directions for test administration followed the TOPL-2 examiner's manual.

The process: The child or adolescent looked at the picture and heard the question before providing an answer that indicated a pragmatic element. The researcher followed all directions provided in the examiner's manual.

Scoring: The child or adolescent was awarded (1) point for correct answers and (0) points for incorrect answers. The scores were then calculated to obtain the raw score.

Preliminary analysis steps: After recording the raw score by calculating the correct and incorrect answers, each raw score was converted to a Pragmatic Language Usage Index and percentile rank using the appendix in the examiner's manual. Standard scores, grades and age equivalents were also recorded after conversion from the raw score.

\section{RESULTS}

SPSS version 17 was used to statistically analyze the data collected in this study. Descriptive statistics were used to obtain means, standard deviations and ranges for certain issues in this study. In addition, frequencies were also used to calculate the descriptive rate of the participating cases and other issues, illustrated in the figures and tables below.

To identify typical and atypical PLI manifestations in individuals with DLD using the Arabic TOPL-2, the first major aim of this study, the tests was administered twice on twelve cases. Table 2 compares the results of the two Arabic TOPL-2 administrations. The results appearing in the means, standard deviations and ranges are not completely consistent. The highest mean in the first administration is 6.1 with $5.8 \mathrm{SD}$ and 18 range for the physical context subcomponent. In comparison, the highest mean in the second administration is 4.6 with 5.7 $\mathrm{SD}$ and 16 range for the same subcomponent. Additionally, the lowest mean value is .25 with $.62 \mathrm{SD}$ and (2) range for the abstractions subcomponent. Conversely, the lowest value mean in the second Arabic TOPL-2 administration is .08 with $2.3 \mathrm{SD}$ and 1 range for the same subcomponent. The attained data resulting from the doubled Arabic TOPL-2 assessment battery are thus similar but not completely consistent.

TABLE 4: Results on PC measures by administration times (means, standard deviations, and ranges)

\begin{tabular}{lllllllll}
\hline TOPL-2 components & $1^{\text {st }}$ time administration & \multicolumn{5}{c}{$2^{\text {nd }}$ time administration } \\
\hline Situational context & $\mathrm{n}$ & mean & $\mathrm{SD}$ & range & $\mathrm{n}$ & mean & $\mathrm{SD}$ & range \\
Physical context & 12 & 2.8 & 2.8 & 9 & 12 & 2.8 & 3.3 & 10 \\
Audience & 12 & 6.1 & 5.8 & 18 & 12 & 4.6 & 5.7 & 16 \\
Discourse context & 12 & 3.8 & 3.8 & 12 & 12 & 3.7 & 4.4 & 13 \\
Topic & 12 & 1.2 & 1.2 & 4 & 12 & 1.5 & 1.4 & 4 \\
Purpose & 12 & 1.7 & 1.8 & 4 & 12 & 1.3 & 2.05 & 6 \\
Semantic context & 12 & 1.3 & 1.5 & 4 & 12 & .83 & 1.4 & 4 \\
V-G cues & 12 & 4.7 & 4.6 & 15 & 12 & 3.7 & 4.4 & 13 \\
Abstractions & 12 & 1.4 & 1.3 & 4 & 12 & 2.0 & 2.1 & 6 \\
Pragmatic E & 12 & .25 & .62 & 2 & 12 & .08 & 2.3 & 1 \\
\hline
\end{tabular}


Figures 1 and 2 illustrate and compare the descriptive ratings of 12 participants with DLD for the two Arabic TOPL-2 administrations. In both pie charts, no participant achieved a rank higher than below average. The highest percentage of participants achieved a descriptive rating of poor, with (over $41 \%$ ) doing so in the first administration and $(50 \%)$ in the second. The highest percentage in Figure 1 is repeated again in the descriptive rating below average, which is significantly less in Figure 2 (16\%). Conversely, the very poor rating in Figure 2 is about twice as frequent as the poor rating in Figure 1. Most participating cases in this study seem to have more atypical than typical PC manifestations, which can be inferred from the descriptive ratings shown in Figures 1 and 2.

\section{Figure 1TOPL-2 Performance-First time Administered}

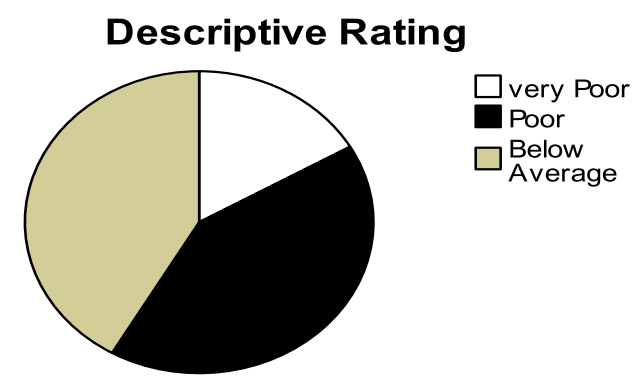

\section{Figure 2 TOPL-2 Performance- Second Time Administered}

\section{Descriptive Rating}

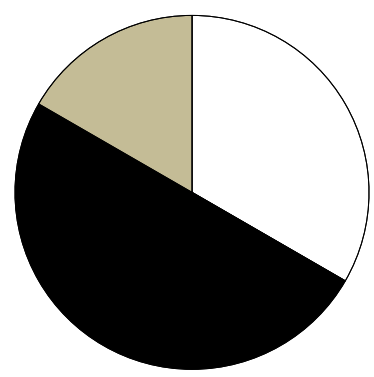

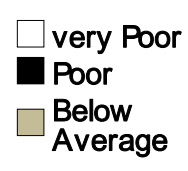

Figures 3 and 4 show the raw scores of 12 participants with DLD obtained from the Arabic TOPL-2 administration using replication logic methodology. The highest and lowest achieved raw scores in both Arabic TOPL-2 administrations are similar. While the raw score for the first administration peaks at 1 and 3 , that for the second peaks at ( 0 and 2 ). The lowest raw score in both figures is 0 ; the frequency of this score is 1 in the first administration and 3 in the second. Another slight difference between the administrations is that the highest achieved raw score is 12 in the first and is less in one score only in the second administration. Participants in this study appear to have serious PC problems, and the slight differences in the results might be attributed to the development of the primary disorders in the participating cases in the one-week gap between the first and second administrations.

Figure 3 First time TOPL-2

\section{Administered- Raw Score}

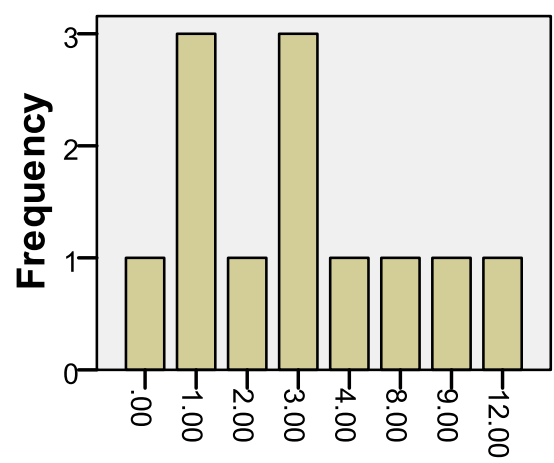

Raw Score

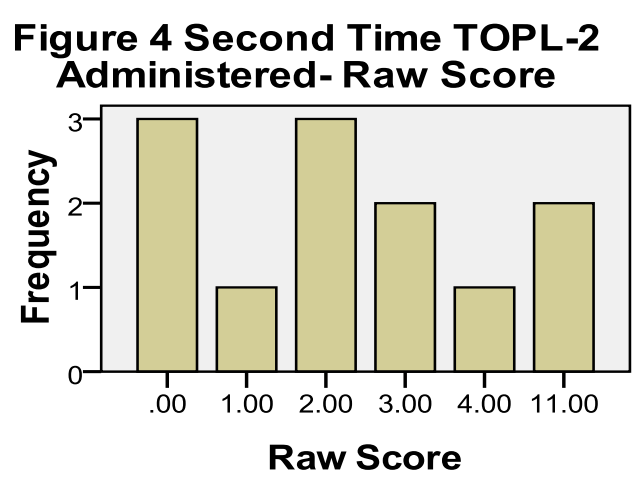

Because verbal communication ability is inexorably related to PC, IQ test results were analyzed for the participating cases. Figure 5 presents the IQ test results of the 12 participants in this study to show the effect of a PLI on IQ ability or vice versa. The descriptive rating of the achieved IQ results varied from low (n: 1), borderline low (n: 1), below average (n: 1), lower average (n: 8) and higher average (n: 1). The most frequent rating is lower average. Therefore, an intricate relationship between the PC and IQ test performance of the participating children and adolescents can be assumed. 


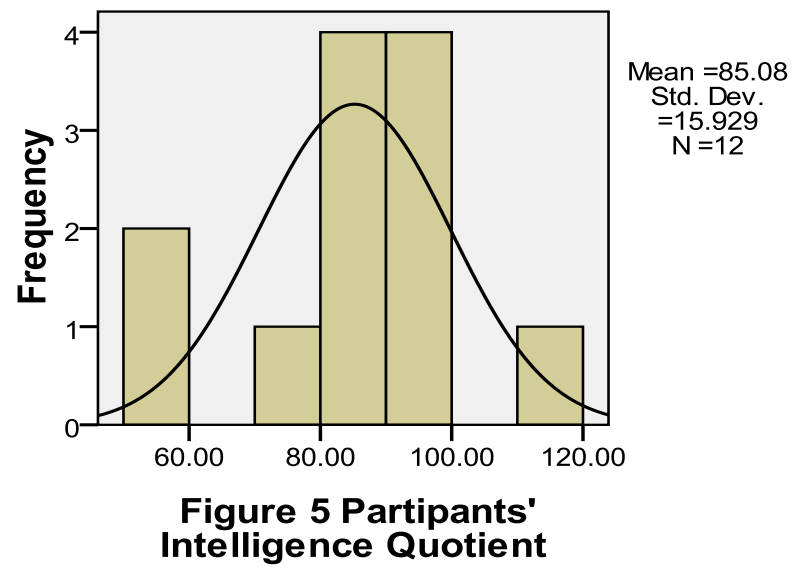

The data obtained after converting the raw scores of all participants into age and grade equivalents using the TOPL-2 examiner's manual, in table 5, indicate that poor PC skills do not only negatively affect linguistic abilities but can also lead to include younger emotional and mental ages and poor scholastic achievement. Converting the raw scores into age and grade equivalents, the real participant ages and grade levels were considerably higher than their TOPL- 2 age and grade equivalents. The real participant ages ranged from 6-12, but their equivalent ages were only in the range of 6 years and 9 months for all cases. The true grade levels, 1-6, strongly differed from the grade equivalents, which ranged from sub-kindergarten 3 to grade 1 . The clear differences between the subjects' equivalent age and grade differences and their real ages and grades presumably affect their school and social lives.

\begin{tabular}{cccc}
\hline \multicolumn{3}{c}{ TABLE 5: Real age, equivalent age and grade (Means \& SDs) } \\
Real Age & $\begin{array}{c}\text { Age Equivalent } \\
\text { Grade } \\
\text { Equivalent }\end{array}$ \\
Mean & 8.33 & 6.00 & .08 \\
Std. Deviation & 2.23 & .00 & .29 \\
Minimum & 6.00 & 6.00 & .00 \\
Maximum & 12.00 & 6.00 & 1.00 \\
\hline
\end{tabular}

The TOPL-2 authors presented pragmatic ability using three main components and seven subcomponents: situational (physical context and audience), discourse (topic and purpose) and semantic contexts (visual-gestural cues, abstractions and pragmatic evaluation). For comparison, table 6 presents the most typical and atypical PLI manifestations of the participating cases. All cases have poor pragmatic skills regardless of manifesting few typical features. The most atypical pragmatic manifestation is the abstractions subcomponent (M: .25, SD: .62), and the most typical is the purpose context subcomponent (M: 4.75, SD: 4.57). Conversely, the least typical pragmatic manifestation among the main components is the situational context, and the least atypical pragmatic manifestation is the discourse context component. The second and third-most typical pragmatic manifestations among the seven subcomponents are physical context and visual-gestural cues. The fourth-most typical pragmatic manifestations are the audience and topic contexts subcomponents. The number five typical pragmatic manifestation is pragmatic evaluation subcomponent. Finally, the most impaired pragmatic manifestations in the participating cases are those related to situational contexts, followed by the semantic context pragmatic elements.

\begin{tabular}{lllllll}
\hline \multicolumn{5}{c}{ TABLE 6: Participants Performance on Arabic TOPL-2 Psycholinguistic Marker: typical and atypical } \\
& $\mathrm{N}$ & Minimum & $\begin{array}{c}\text { manifestations } \\
\text { Maximum }\end{array}$ & Sum & M & SD \\
& 12 & 0 & 4 & 14 & 1.17 & 1.19 \\
Physical Context & 12 & 0 & 5 & 20 & 1.67 & 1.82 \\
Audience Context & 12 & 0 & 5 & 16 & 1.33 & 1.50 \\
Topic Context & 12 & 0 & 15 & 57 & 4.75 & 4.57 \\
Purpose Context & 12 & 0 & 4 & 17 & 1.42 & 1.31 \\
V-G Cues & 12 & 0 & 2 & 3 & .25 & .62 \\
Abstractions & 12 & 0 & 7 & 26 & 2.17 & 2.40 \\
Pragmatic Eva. & 12 & 0 & 9 & 34 & 2.83 & 2.85 \\
Sit. Context & 12 & 0 & 18 & 73 & 6.08 & 5.88 \\
Discourse Context & 12 & 0 & 12 & 46 & 3.83 & 3.81 \\
Semantic Context & 0
\end{tabular}


A minor aim of this study was to identify any differences in PLI severity among four forms of DLD: SLI (n: 6), DLD (n: 4), DLAPh (n: 1) and LD (n: 1), illustrated in table 7. The first three columns show the different forms of DLD and their raw scores. The last four columns present the cases according the language impairment that resulted in a PLI. As shown in the table, only those who have DLDs caused by mental retardation (MR) have very poor achievement in the Arabic TOPL-2 test. In other cases, participants have either mild PLIs, as in (5 \& 8: SLI, 6 \& 9: DLD, and 10: LD), or severe, as in (1, 2, 4, 7: SLI, 3: DLAPh). A PLI's severity seems to be controlled by the type of primary disorder, whether congenital (developmental dysphasia) or neurolinguistic (acquired child aphasia).

TABLE 7: Degree of effect of the primary impairment on pragmatic competency

\begin{tabular}{|c|c|c|c|c|c|c|}
\hline $\begin{array}{l}\text { Case } \\
\text { No. }\end{array}$ & Impairment & Raw score & $\begin{array}{l}\text { Case } \\
\text { No. }\end{array}$ & Impairment & $\begin{array}{l}\text { Raw } \\
\text { score }\end{array}$ & Descriptive rating \\
\hline 1 & SLI & 0 & 1 & SLI & 0 & Poor \\
\hline 2 & SLI+LD & 9 & 2 & SLI & 9 & Poor \\
\hline 3 & DLAPh & 1 & 4 & SLI & 12 & Poor \\
\hline 4 & SLI+LD & 12 & 5 & SLI & 8 & Below average \\
\hline 5 & SLI & 8 & 7 & SLI & 1 & Poor \\
\hline 6 & DLD & 4 & 8 & SLI & 3 & Below average \\
\hline 7 & SLI & 1 & 6 & DLD & 4 & Below Average \\
\hline 8 & SLI & 3 & 9 & DLD & 3 & Below average \\
\hline 9 & DLD+SLI & 3 & 11 & DLD & 1 & Very poor \\
\hline 10 & $\mathrm{LD}$ & 3 & 12 & DLD & 2 & Very poor \\
\hline 11 & DLD+MR & 1 & 3 & DLAPh & 1 & Poor \\
\hline 12 & DLD+MR & 2 & 10 & $\mathrm{LD}$ & 3 & Below average \\
\hline
\end{tabular}

\section{DISCUSSION}

There were 12 participants in this study: 6 with SLI, 4 with DLD due to mental retardation, 1 with DLAPh and 1 with LD. After examining the medical reports and case records and analyzing the results of the psycholinguistic battery, the cases seemed similar despite the differences in primary disorder.

For instance, case 1 could utter 3-4 word sentences with good semantics and affected syntax (sex inflection, verb tense, negation and plurals) and multiple phonological errors. According to the medical file, the child has normal hearing sensitivity, normal development milestones and normal mentality. Generally, neither ADHD signs nor autistic and behavioral traits were observed. The first evaluation revealed that the child is cooperative, with good eye contact and imitation ability. His receptive abilities are good, as he could recognize most different semantic groups. Regarding his expressive abilities, he could utter 3-4 word sentences with good semantics and affected syntax. The final evaluation revealed that he could utter long sentences with good semantics. During the evaluation, the child used only short sentences and must repeat or modify the questions to continue his speech to form long sentences. Finally, the child's relationship with his family seems to be mostly negative, which again hinders the development of his pragmatic skills. He was the third child of six, but he was uncooperative, stubborn and refused to repeat after his mother, though he responds sometimes.

Conversely, case 2 received regular therapy sessions to increase the length of her sentences and correct both syntactic and phonological errors. She has normal peripheral hearing sensitivity and could utter long sentences with good semantics and good syntax with misarticulation of certain phonemes. In the context of her family, she is cooperative but shy and prefers to be alone.

Case 3 was the elder of two children. She was cooperative and attentive, with good response during evaluation. She had good behavior and relationships with her family, relatives and friends. She had good imitation ability and tries to correct her phonatory errors. She responded well to instructions and orders. Her development history results and development milestones were normal. However, her medical records indicated an illness in early childhood: febrile convulsions starting at 1 year of age and ending at 3 years of age. According to her mother, the child could utter long sentences; during evaluation, she mainly uses 4-5 word sentences with good semantics but affected syntax regarding plurals, negation and verb tense with multiple phonological errors.

The main complaint of case 4's mother was poor scholastic achievement, poor reading and writing abilities and the inability to utter certain phonemes correctly. His developmental history and development milestones were normal. He could utter long sentences with good semantics and syntax, with misarticulation of $/ \mathrm{k} /$ and /g/ sounds. The child preferred to utter short sentences with simple syntax and laugh instead of continuing to answer questions. He was the first child of three and cooperative with good familial relationships, but he refuses to respond to his mother's corrections. 
Case 5 was an uncooperative child, shy and stubborn. According to his mother, he could utter long sentences when he wants to. During the sessions, he used only 2-3 word sentences with good semantics and affected syntax (sex inflection, verb tense, negation and plurals) and multiple phonological errors. He spoke rapidly, which negatively affects his speech intelligibility. According to the medical file, the child had normal hearing sensitivity, normal development milestones and normal mentality. Neither ADHD nor autistic traits were observed. There was also no abnormal behavior detected, except being uncooperative and slightly shy. His mother reported that he is stubborn and refuses to respond to her at home when she asks him to slow and correct his speech. The results of his first evaluation revealed that he was uncooperative with poor eye contact and poor imitation ability. His receptive abilities were good, as he could recognize most of the different semantic groups. Regarding his expressive abilities, he could utter 2-3 word sentences with good semantics. After the last evaluation, he could utter long sentences with good semantics. During evaluation, the child used only short sentences and must repeat or modify the questions to form long sentences. He was the third of six children.

Case 6 suffered from repeated convulsion attacks twice daily during the first month after birth, controlled with the treatment. During evaluation, he could utter 2-3 word sentences with good semantics, affected syntax and multiple phonological errors that affected his speech intelligibility. According to the medical file, the child had normal hearing sensitivity and delayed development milestones. He started to utter his first word at 2 years of age and first sentence at 3 years and 6 months. No abnormal behavior could be detected, except uncooperative and slightly shy behavior. His first evaluation revealed that he was uncooperative with poor eye contact and imitation ability. His receptive abilities were good, as he could recognize most different semantics groups. Regarding his expressive abilities, he could utter 2-3 word sentences with good semantics and multiple phonological errors. At the last evaluation and as verified by his mother, his language abilities improved greatly: he could utter long sentences with good semantics. During evaluation, the child uses only 3-4 word sentences with fair syntax and phonological errors. He was the fifth child of eight and generally shy.

Case 7 was cooperative in the first evaluation, with good eye contact and response during evaluation. According to the medical file, the child had normal hearing sensitivity and development milestones. She started to utter her first word at the age of 1 and her first sentence at 2 y $6 \mathrm{~m}$. No abnormal behavior was detected, although she was slightly shy. She could utter 3-4 word sentences with good semantics and affected syntax with phonological errors, which affected her speech intelligibility. The results of the last evaluation indicated that she could utter long sentences. Regarding her familial relationships, she is a cooperative child.

Case 8 is similar. The first evaluation revealed normal vaginal delivery of a low-birth-weight preterm infant. The child had normal hearing sensitivity, development milestones and mentality. Generally, neither ADHD nor autistic and behavioral traits were observed. During evaluation, the child could utter 3-4 word sentences with good semantics, affected syntax and multiple phonological errors, affecting her speech intelligibility. The child was cooperative with fair eye contact and imitation ability. Her receptive abilities are good, as she could recognize different semantics groups. Regarding her expressive abilities, she could utter 3-4 word sentences with good semantics, affected syntax and multiple phonological errors affecting his speech intelligibility. The results of the last evaluation showed strong improvement in her linguistic abilities. During the last evaluation, she could utter 4-5 word sentences with fair syntax and phonological errors. She was the $5^{\text {th }}$ of 5 children and has good relations with her family.

Case 9 could utter 3-4 word sentences with good semantics and affected syntax (sex inflection, verb tense, negation and plurals) and multiple phonological errors. According to the medical file, the child has normal hearing sensitivity, development milestones and mentality. Generally, neither ADHD nor autistic traits were observed. No abnormal behavior was detected, except shyness. The first evaluation revealed that she is cooperative with good eye contact and imitation ability. Her receptive abilities are good, as she could recognize most different semantics groups, except colors. Regarding her expressive abilities, she could utter 2-3 word sentences with good semantics with affected syntax. In the last evaluation, she could utter 3-4 word sentences with good semantics and affected syntax. She is the sixth child of seven and is cooperative but shy. She sometimes refuses to respond to commands and follow instructions.

Like case 9, case 10 could utter long sentences with good semantics and fair syntax and phonology. According to the medical file, the child has normal hearing sensitivity, development milestones and mentality. Generally, neither ADHD nor autistic and behavioral traits were observed. The first evaluation revealed that the child is cooperative with good eye contact and imitation ability. His receptive abilities are good, as he could recognize different semantic groups. Regarding his expressive abilities, he could utter long sentences with good semantics and fair syntax. He sometimes uses 4-5 word sentences and pauses for a few seconds before completing his sentence. In the last evaluation, he could utter long sentences with good semantics. During evaluation, the child only uses simple sentences and must have the questions repeated to continue his speech to form long sentences. He is the fifth child of nine and is cooperative but sometimes refuses to repeat after his mother.

Finally, cases 11 and 12 identical (twins) are the second and third children of three. They are enrolling in a school for mentally handicapped children. They are generally cooperative but shy. They have been 
diagnosed as pre-term low birth weight infants with delayed development milestones. In the psychometric evaluation, they showed poor attention skills. The children could utter 4-5 word sentences with good semantics and fair syntax. They mainly use two-word sentences and they must have the questions repeated to continue their answer. Their relation with family is good, especially with each other; they depend on one another.

Concerning the identified typical and atypical PLI manifestations, the differences and similarities between and among the cases varied significantly. Generally, the results of the two test administrations indicated that the most typical PLI manifestations for all cases are the semantic context elements. Conversely, the most atypical PLI manifestations are the discourse context elements. Semantic context refers to 'the meaning of a communication and to the aspects of language that are used to convey the concepts or ideas' (Phelps-Terasaki and Phelps-Gunn, 2007, p. 4). Furthermore, the semantic context has three subcomponents according to the TOPL-2 model: visual-gestural cues, abstractions and pragmatic evaluation (ibid). The discourse context refers to 'the function of the language in individual uses in a communication and the relative effectiveness of different methods of conveying the particular intent' [1, (p. 3)]. Unlike the semantic context, which has three subcomponents, the discourse context has only two subcomponents according to the TOPL-2 model: topic and purpose (ibid).

Comparing the twelve participants' typical and atypical PLI manifestations, cases 1, 3 and 11 have the same level of both the most typical and atypical manifestations, and the results prove that each participant has severe PLIs. Cases 2, 4, 5, 6, 8, 9, and 10 differ from the above cases. These cases seemed similar in the most typical and atypical PLI manifestations: the most typical PLI manifestations for each case are discourse context elements, and the most atypical are the situational and semantic context elements. The situational context pragmatic component 'represents the physical environment in which discourse takes place and the characteristics of the audience to which conversation is directed' [1, (p. 2)]. The remaining cases, 7 and 12, have different indications about the most typical and atypical PLI manifestations. In case 7, both situational and discourse contexts elements are in the same level and form the most atypical manifestations, whereas discourse context elements form the most typical manifestations. In case 12, semantic context elements are the most atypical PLI manifestations, but both situational and discourse contexts are the most typical ones.

\section{CONCLUSION}

This paper aimed to identify both atypical and typical PLI manifestations in individuals with different types of delay language disorders using the Arabic TOPL-2. This paper claimed, however, that using the Arabic TOPL-2 can identify and measure the most atypical and typical manifestation of each of the twelve participating cases. Both the statistical results and discussion of the collected data have clearly consolidated the claimed hypothesis rather than refuting it. The used psycholinguistic marker could give a proposed order for the most atypical or typical PLI manifestations: situational, semantic and discourse contexts, for the pragmatics components. The pragmatics subcomponents were ordered according to the most atypical: abstractions, pragmatic evaluation, topic, audience, visual-gestural cues, physical context, and purpose. Moreover, the two test administrations indicated that the results were not completely consistent; instead, they were relatively consistent. Last, the results of the achieved scores in the TOPL-2 measure also indicate that PLI severity is strongly influenced by the type of the primary impairment. In this study, children with mental retardation and preterm births demonstrated more severe PLIs than other cases with only SLI, LD or DLD. Such results have led to a proposed conclusion that disorders of the biolinguistic type (dysfunction or disturbance of some linguistic genes) could inevitably result to severe PLIs, as in the three cases in this study.

\section{CLINICAL IMPLICATIONS}

A complementary claim of the primary hypothesis of this study was that designing a rehabilitation program or treatment plan based on the identification of the most atypical and typical PLI manifestations would improve the level of individuals identified as pragmatically impaired. Although this claim was not tested in this study, it was not refuted. As a result, this study has clinical implications for speech and language pathologists, interventionists and researchers in clinical linguistics, biolinguistics, neurolinguistics and psycholinguistics. The above complementary claim was not refuted because the proposed treatment plans for each participant in this study neither included nor named pragmatic competency as a language component or skill.

The proposed plans are summarized in the following points: 1) detailed family counseling to provide extended, corrective feedback and phonological and syntactic error correction; 2) regular weekly therapy sessions (3-9) to increase sentence length, correct syntactic and phonological errors, slow the speech rate to improve speech intelligibility, correct impaired phonemes, and improve reading and writing abilities; 3 ) counseling to improve reading ability; 4) regular therapy sessions to correct misarticulation and improve learning abilities, wherein mothers were instructed to let their children choose a story and read it aloud, summarize the story verbally and in writing; and listen to the mother's feedback; 5) direct therapy sessions to improve reading and writing abilities, with a focus on improving scholastic achievement; and 6) assessing 
psycholinguistic abilities using the Illinois test to confirm diagnoses regarding learning disabilities and detect weaknesses that affect scholastic achievement.

Consequently, using the results of this study and similar studies, all speech-language pathologists and interventionists can determine exactly which components and subcomponents require intensive rehabilitation program(s) and which require lower intensity rehabilitation program(s).

Researchers can also investigate the following points based on this study: 1) clinical linguists can stress the importance of using specific psycholinguistic markers to identify each language component more strongly, 2) biolinguists can investigate whether it is true that certain genes represent pragmatics in the brain, which make PLI occur when these unknown genes are injured, as in the case of the twins and preterm children in this study, 3) neurolinguists can use imaging techniques to investigate the conclusion in this study that a certain area in the brain is responsible for pragmatics components, and 4) psycholinguists can use the TOPL-2 model to determine whether an order can be drawn in normal individuals in early- and late-acquired pragmatic components and subcomponents.

\section{LIMITATIONS OF THE STUDY}

Two limitations in this study were noted. First, consistency in a subject's primary disorders was unachievable. This impossibility governed the available cases in the hospital at the time and during the allowed research period. Second, it is difficult to consolidate the claim that using a rehabilitation program designed based on the results of this study would be more effective than the rehabilitation programs previously used and designed in the Communication and Swallowing Disorders Unit (CSDU) where this research was performed. Future work should use a consistent sampling method so that the comparison between and among cases would be more plausible. Furthermore, testing the correlation between the findings of the study and the effectiveness of the designed rehabilitation program should be considered more thoroughly.

\section{Acknowledgements}

The first author would like to sincerely thank Professor Mohammed Ziad Kebbe for his motivation and psychological support. The authors would like also to thank the IRB committee at King Khalid University Hospital, College of Medicine, KSU, Riyadh, Saudi Arabia for issuing both the IRB and consent forms for the original research. Many thanks are also due to those in the Communication and Swallowing Disorder Unit (CSDU) and the Research Chair of Voice, Swallowing, and communication Disorders, King Abdulaziz University Hospital, College of Medicine, KSU. The unforgettable efforts of the secretary of the IRB committee are also highly appreciated and deserve acknowledgment.

\section{REFERENCES}

[1] Phelps-Terasaki, D., \& Phelps-Gunn, T. TOPL-2 Test of Pragmatic Language: Examiner's Manual (2 ed.). (Shoal Creek Boulevard, Austin, Texas: Pro-ed Inc, 2007).

[2] Kent, R. D. (Ed.). The MIT Encyclopedia of Communication Disorders. (Cambridge, Massachusetts, London, England: A Bradford Book The MIT Press, 2004).

[3] Bavin, E. L. (Ed.). The Cambridge Handbook of Child Language. (Cambridge: Cambridge University Press, 2009).

[4] ASHA. American Speech-Language-Hearing Association (ASHA): USA, 1997-2012. Retrieved 11 10, 2010, from American Speech-Language-Hearing Association: http://www.asha.org

[5] Norris, J., \& Hoffman, P. Whole Language Intervention for School-age Children. (San Diego: CA Singular, 1993).

[6] Verschueren, J., Ostman, J.-o., Blommert, J., \& Bulcaen, C. Handbook of Pragmatics. (Amsterdam, Philaelphia: John Benjamins Publishing Company, 2002).

[7] Perkins, M. Pragmatic Impairment. (Cambridge: Cambridge University Press, 2007).

[8] Perkins, M. R. The scope of pragmatics disability: A cognitive approach. In N. Muller, Pragmatics in speech and language pathology: studies in clinical applications. (Amsterdam, Philadelphia: John Benjamins Publishing, 2000) 7-23.

[9] Noveck, I. A., \& Sperber, D. (Eds.). Experimental Pragmatics. (New York: Palgrave Macmillan Ltd, 2004).

[10] Fabbro, F., \& Asher, R. E. (Eds.). Concise encyclopedia of language pathology. (Amsterdam - Lausanne - New York - Oxford Sahnnon - Singapore - Tokyo: Elsevier, 1999).

[11] Kim, O. H. Language characteristics and social skills of children with attention deficit hyperactivity disorder. Ed.D. dissertation, Peabody College for Teachers of Vanderbilt University, United States -- Tennessee. Retrieved October 13, 2010, from Dissertations \& Theses: Full Text.(Publication No. AAT 9929118), 1999.

[12] Kim, O. H., \& Kaiser, A. P. Language Characteristics of Children with ADHD. Communication Disorders Quarterly, 2000, 154165.

[13] Adams, C. Practitioner Review: The assessment of language pragmatics. Journal of Child Psychology and Psychiatry, 43 (8), , 2002, 973-987.

[14] Busari, J. O., \& Weggelaar, N. M. How to investigate and manage the child who is slow to speak: Clinical review, 2004. Retrieved 10 10, 2010, from Google, BMJ: http://www.bmj.com/content/328/7434/272.full.pdf+html

[15] Ingersoll, B., Dvortcsak, A., Whalen, Ch., \& Sikora, D. The Effects of a Developmental, Social-Pragmatic Language Intervention on Rate of Expressive Language Production in Young Children With Autistic Spectrum Disorders. Focus on Autism and Other Developmental Disabilities, 20(4), 213-222, 2005. Retrieved October 13, 2010, from ProQuest Science Journals. (Document ID: 947112811). 
[16] Volden, J., Coolican, J., Garon, N., White, J., \& Bryson, S. Brief Report: Pragmatic Language in Autism Spectrum Disorder: Relationships to Measures of Ability and Disability. Journal of Autism and Developmental Disorders, 388-393, 2009. Retrieved from: http://search.epnet.com, at 01/11/2010.

[17] Volden, J., \& Phillips, L. Measuring Pragmatic Language in Speakers With Autism Spectrum Disorders: Comparing the Children's Communication Checklist-2 and the Test of Pragmatic Language. American Journal of Speech - Language Pathology (Online), 19(3), 204-212A, 2010. Retrieved October 13, 2010, from ProQuest Medical Library. (Document ID: 2151665871).

[18] Alduais, A. M. The Use of Two Pragmatic Language Tests to Identify Typical and Atypical Manifestations of PLI: A Large-scale Study on Saudi Children and Adolescents with Developmental Dysphasia. Unpublished MA thesis, King Saud University (KSU), Riyadh, Saudi Arabia, 2013.

[19] Alduais, A. M., Shoeib, R. A., Al-Hammadi, F. S., \& Al-Malki, K. H. Testing the Usability of an Arabic Version of TOPL-2 in Measuring Pragmatic Language Impairment in Children and Adolescents with Developmental Dysphasia. International Journal of Linguistics (IJL), IV (2), 2012, 193-214.

[20] Alduais, A. M., Shoeib, R. A., Al-Hammadi, F. S., Al-Malki, K. H., \& Alenezi, F. H. Measuring Pragmatic Language in Children with Developmental Dysphasia: Comparing Results of Arabic Versions of TOPL-2 and CELF-4 (PP and ORS Subtests). International Journal of Linguistics (IJL) , 4 (2), 2012, 475-494.

[21] Yin, R. K. Case Study Research: Design and Methods. (Thousand Oaks, London, New Delhi: Sage Publications, 2003). 